\title{
SNP Analysis, Genome Wide Association Studies and Pharmacogenetic implications of Acute Myocardial Infarction
}

\author{
Jayasree Ganugapati, PhD \\ Department of Biotechnology \\ Sreenidhi Institute of Science \\ and Technology, \\ Telangana, India - 501301
}

\author{
Prakhyat Mathur \\ Department of Biotechnology \\ Sreenidhi Institute of Science \\ and Technology, \\ Telangana, India - 501301
}

\author{
Sudhira Chamarty \\ Department of Biotechnology \\ Sreenidhi Institute of Science \\ and Technology, \\ Telangana, India - 501301
}

\author{
Supriya Krishna \\ Department of Biotechnology \\ Sreenidhi Institute of Science and Technology, \\ Telangana, India - 501301
}

\begin{abstract}
Coronary Heart Disease is a condition wherein there is a buildup of plaque in the coronary artery that creates a blockade reducing the supply of oxygen to the myocardial cells. As the muscle cells begin to undergo necrosis, the body is said to be in a state of Mycocardial Infarction. Even though the condition of Myocardial infarction (MI) is extensively associated with lifestyle, recent evidence has indicated the role of genetics in the widespread occurrence of disease. Linkage Disequilibrium study of the genes associated with myocardial infarction was the foundation for a more advanced study of pharmacogenetic implications for drug efficacy and toxicity. The study aided in identification of new drug targets and in the mapping of genetic associations in various populations that hence contribute to personalized medicine. In the present study, the authors performed genome-wide association studies to compare the genes associated with Myocardial Infarction (ACE, MIA3, GATA4, ESR1) in five populations. Linkage Disequilibrium (LD) analysis was performed to understand the association of various SNP's present on these genes. Post-retrieval of SNP genotype data of various populations from NCBI's 1000genomes browser, an LD plot was constructed using an online LD Link LD Analysis tool developed by National Cancer Institute. The results have indicated a dense map of single nucleotide polymorphisms (SNPs) which were linked to Myocardial Infarction and SNPs that are co-inherited in different populations. Further analysis of SNP's using PharmGKB database suggested the SNP's influence on drug therapy of prescribed drugs for Myocardial Infarction like Fluvastatin, Raloxifene, Letrozole, Warfarin, and Vancomycin.
\end{abstract}

\section{General Terms}

SNP Analysis, Linkage, Genome Wide Association Studies, Pharmacogenetic Implication Study.

\section{Keywords}

Linkage Disequilibrium, SNP Analysis, Myocardial Infarction, Pharmacogenomics.

\section{INTRODUCTION}

Coronary Heart Disease (CHD) is a medical condition that develops over time with buildup of waxy plaque inside the coronary arteries. Subsequent buildup of plaque could rupture the arteries walls leading to the formation of a blood clot. If the clot reaches a large enough size, it could completely block blood flow through the artery and lead to a heart attack. In a heart attack, there is an abrupt shortage of blood flow to the heart. This leads to considerable tissue damage in the heart muscle cells that undergo necrosis in the prolonged condition of ischemia. Without any blood supply, the inherent cellular repair mechanisms for the heart muscle cells fail and are unable to maintain homeostasis. The muscle cells that stop functioning die because of necrosis and this state is referred to acute myocardial infarction. It is the state where the myocardial cells begin to die due to loss of repair function as the supply of nutrients through blood is hindered. Being one of the largest causes of mortality worldwide, a detailed study of the genes associated with the occurrence of the disease and their linkage was investigated.

\subsection{Genes considered for the study}

The genes that were considered for the study were found to play a role in the occurrence of Acute Myocardial Infarction.

\subsubsection{ACE:}

The gene codes for an enzyme that is responsible for converting Angiotensin from an inactive form to an active one. Abbreviated as ACE, it is linked to cases of Acute Myocardial infarction. The enzyme, in the body, catalyzes the conversion of Angiotensin I into an active peptide Angiotensin II. [1] The active protein, Angiotensin II plays an important role in the maturation of renal tissue as it stimulates the constriction of blood vessels and secretion of aldosterone. This mechanism controls the blood pressure [2] and fluid electrolyte balance. Certain studies have reported findings that have linked a $287 \mathrm{bp}$ 'ALU' repeat sequence in this gene to cardiovascular conditions. [3][4]

\subsubsection{MIA3:}

The gene MIA3 encodes for a protein MIA3, a member of the Melanoma inhibitory activity protein family. The protein is also referred to as Transport and Golgi organization protein 1 (TANGO1). It is an enzyme that is involved in the packaging of molecules like Collagen VII into COPII vesicles out of the Endoplasmic Reticulum. [5] Transport of collagen indicates that the enzyme is involved in osteogenesis. Recent evidence has indicated that the gene coding for MIA3/TANGO1 
contains one of 27 loci that are listed under an increased risk of coronary artery disease. [6]

\subsubsection{GATA4:}

Encoding for a member of the protein family of zinc-finger motif transcription factors that recognize and bind to the sequence 'GATA' on promoters of many genes, the gene GATA4 is involved in signal transduction. The protein is involved in regulation of the transcription of genes related to embryogenesis, normal testicular development, and maturation of myocardial cells. [7] Further evidence of the gene's relevance is that certain mutations in this gene are linked to congenital heart disease, hypoplasia of the ventricular myocardium, and cardiac septal defects. [8] Gene expression studies for the gene have found that alterations in the expression have been linked to several cancers. [9]

\subsubsection{ESR1}

The gene codes for an estrogen receptor that plays a role in transcription regulation. Once the respective ligand binds to the receptor, through subsequent signal transducers, the domains coding for genes that are associated with DNA binding proteins and other transcription activators, are expressed. Even though the receptor's products are mainly associated with sexual development, there has been evidence linking the receptor to function in other tissues such as bone [10]. Alterations in gene expression have indicated that the receptor is also involved in the development of certain cancers [11], osteoporosis, and heart disease [12] [13].

The aforementioned genes perform their function in adequacy in healthy individuals. However, the function of the gene could be altered with mutations, like Single Nucleotide Polymorphisms (SNPs) that have been widely studied for the past few decades. Their importance in diseases and recently in influencing disease risk and effectiveness of drug therapy inspired this study. The objective of this study is to correlate genetic linkage and inheritance patterns between SNP variants seen in the genes associated with acute myocardial infarction across various populations of the world. This is possible through genome-wide association studies of a set of genetic variants in different individuals from various populations of the world to investigate the possibility of a variant's association with disease. This information can be used to detect, treat, and prevent a majority of the human diseases including cancers, diabetes, heart disease, etc [14]. The phenomenon of two genes, which are close together, being inherited, is known as Linkage. Linked genes are inherited together to the next generation without any or fewer chances of recombination. Linkage Disequilibrium is one such phenomenon wherein there exists a non-random association between two alleles at different loci such that they are inherited together to the following generation. These two alleles are inherited together more commonly than expected. The alleles which are said to be in Linkage Disequilibrium are correlated and the degree of correlation is inversely proportional to the distance between the alleles. [15] In this study, SNP variants were considered as markers instead of alleles to investigate the patterns of Linkage Disequilibrium. A thorough Linkage Disequilibrium study offers a solid foundation for a pharmacogenetic implication study wherein the SNP variants are investigated to check for classes of drugs that are influenced by the SNP across evidence collected from pharmacological research.

\section{METHODOLOGY}

\subsection{SNP Genotype Data Retrieval}

The aim of researchers behind the 1000 Genomes Project in 2008 was to build an entire database that would feature genomic data from individuals of various populations that would aid in the study of genetic variation across populations. Data on offer from 1000genomes is a key asset to advanced research in pharmacogenetics, bioinformatics, and medicine. [16] For this study, EBI's 1000genomes browser hosted on National Center for Biotechnology Information (NCBI) website was used. SNP genotype data for the four genes shortlisted for the study, aggregated for all sample populations was retrieved. The list of SNPs was then subjected to Linkage Disequilibrium analysis to investigate into the association studies of SNP variants and investigate into the degree of Linkage Disequilibrium.

\subsection{Linkage Disequilibrium Analysis}

LD link (version 2.0) is a complete package of interactive web applications that can be used for analyzing the phenomenon of linkage disequilibrium in a varied list of population groups from a given set of SNP genotype data. [17]. The LD link LD matrix application is used to generate an interactive heat map matrix of pair wise linkage disequilibrium statistics for a population group.

For each LD analysis, specific reference populations were used among the 5 different populations African (AFR), Mixed American (AMR), East Asian (EAS), European (EUR), and South Asian (SAS) available in LD Link.

The LD result is generated in two formats, an LD heat map plot that is used for preliminary analysis of the degree of linkage disequilibrium by the density of the color on the heat map plot that displays both haplotype counts and allele frequencies for two query variants. The results can also be exported as a statistical data table, containing the calculated metrics for linkage disequilibrium that include the $\mathrm{D}$ prime (D') and R-square (R2) values that are calculated using goodness-of-fit statistical tests for deviations of expected frequencies that are sensitive to allelic frequencies. Perfect Linkage Disequilibrium exists in a condition wherein the haplotype frequencies are closer to 1 i.e. both the D' and R2 values are closer to 1 . As R2 is sensitive to allelic frequencies, an SNP association that shares a higher D' value but has a low $\mathrm{R} 2$ is in imperfect linkage disequilibrium as it is associated but the occurrence of that respective allele in a given population is very rare. SNP variant associations for a particular gene that have high LD values throughout the sample populations were said to be in linkage disequilibrium in the sample populations and were subsequently shortlisted for the pharmacogenetic implication study.

\subsection{Pharmacogenetic Implication Study}

Pharm GKB, an abbreviated version of Pharmacogenomics Knowledgebase is an online database that is supported by the National Institute of Health and the Pharmacogenomics research network. Information about the pharmacogenetic study related to the impact of human genetic variation on drug efficacy and toxicity collected from ongoing clinical trials is available. Essentially, the data collected from the database can help in analyzing how the changes in an individual's genetic makeup influence drug therapy. [18] From the list of genes, the genes of interest were selected and their Pgx research was investigated. In the list of SNPs generated, select SNP variants that exhibited high LD are selected and the chemical/drug therapy influenced was studied. The SNP 
variant is one of the primary factors in rendering the drug therapy ineffective in prescriptions and may even lead to drug toxicity in the system.

\section{RESULTS AND DISCUSSION}

LD studies are carried out by employing certain satellite markers for identification, they can be either be microsatellite polymorphisms or as in the case of the present study, Single Nucleotide Polymorphisms. Additional factors like mode of inheritance of the disease, age of the disease-causing mutation(s), and recombination distance between the marker and the disease loci play a major role in affecting the calculation of LD. Considering the example wherein the microsatellite marker has a high rate of mutation, the LD would be extremely low. This is seen more in markers with less mapped distances as the recombination rate and mutation rate is almost similar. This is how an elevated mutation rate affects the reliability of an LD study. This would result in a decrease of LD unless a disease-causing mutation was to arise on a chromosome that would create a new microsatellite allele. For this reason, of the two types of markers, use of haplotypes that contain multiple SNPs enhances the capability of investigating LD.

\subsubsection{Interpretation of an LD Heatmap:}

The sole purpose of analyzing an LD heatmap is to investigate whether any given two SNPs are in Linkage disequilibrium. Consider the generated LD heatmap plot in Fig. as an example, the blocks in the heatmap table generated are known as haplotype blocks. They represent the haplotype of a particular SNP variant. The heatmap plot is a matrix that consists of an equal number of rows and columns that are equal to the number of query SNP variants. In the matrix, to analyze the degree of LD for two given SNP's, the resultant haplotype block achieved by tracking down of the first SNP variant block in the respective column and the second SNP variant block in the respective row or vice-versa indicates the degree of LD.

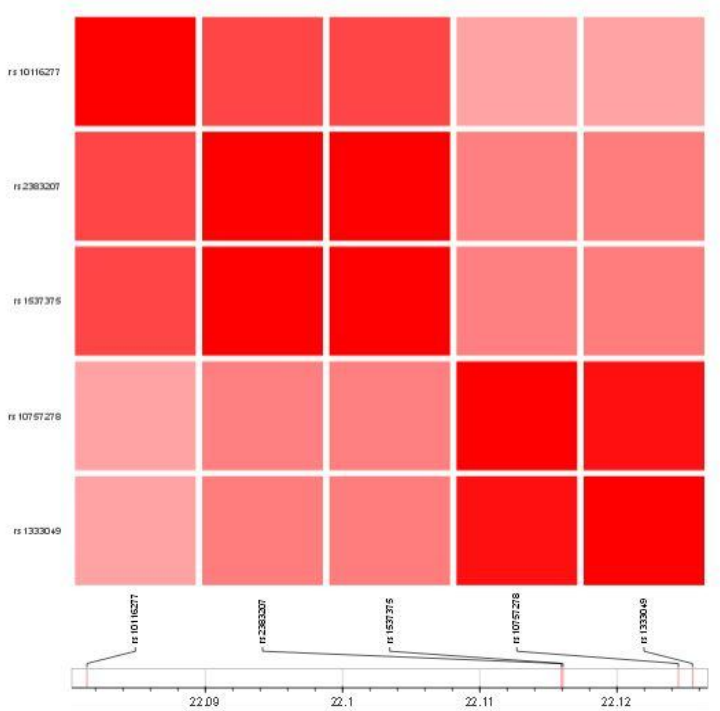

Figure 1: LD Heatmap Plot generated using LDmatrix module of LDLink
The density of the color in the haplotype block indicates the degree to which the given two SNP's are in Linkage Disequilibrium. Alternatively, the data used by the module to plot the heatmap matrix is also available in a statistical tabular format that consists of four columns, the first two being the loci of the two SNP variants being considered, while the third column is the D' value, and the fourth column features the R2 value. The highest value of D' and R2 closest to 1 indicates high LD.

\subsubsection{The parameters of statistical analysis of LD:}

1. L1 and L2 are loci of the two SNP variants being considered for LD investigation

2. D prime $\left(\mathrm{D}^{\prime}\right)$ - It is the normalized value of a parameter that is an indicative of allelic segregation for two given genetic variants (here SNP variants). The value of $\mathrm{D}^{\prime}$ ranges from 0 to 1 , where a value of 0 indicates no linkage of alleles, and a value of 1 indicates a strong linkage of alleles.

3. R-square (R2) - This is an LD measure for correlation of alleles for two given genetic or SNP variants. The value of R2 ranges from 0 to 1 wherein, the value of 0 indicates that the alleles are independent and 1 indicates that there is a high degree of correlation. As R2 is sensitive to allelic frequency, it is not an accurate measure independently. However with D', these two LD measures serve as accurate evidence for Linkage Disequilibrium.

\subsubsection{LD results for gene ACE:}

The LD heatmap matrix plots for ACE gene for different populations are given below. Common to all the sample populations of the present study, two SNP variant associations were noticed. SNP variants rs4331 and rs4341 were noticed to be in high LD, and SNP variants rs4331 and rs4344 were noticed to be in high LD. Even though there were certain SNP variants that were in high LD in a few populations, they did not show a similar degree of LD in other populations and were hence not considered for the pharmacogenetic implication study.

\subsubsection{LD results for gene GATA4:}

The LD heatmap matrix plots for GATA4 gene for different populations was studied and common to all the sample populations of the present study, a single SNP variant association was noticed. SNP variants rs4841588 and rs2645400 were noticed to be in high LD with each other in all sample populations. 


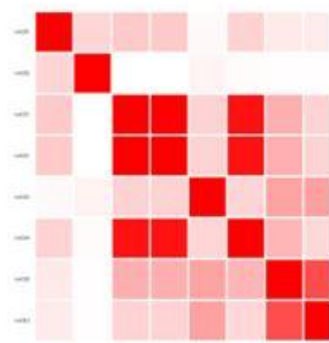

AFR

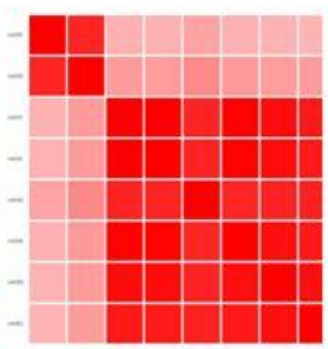

AMR

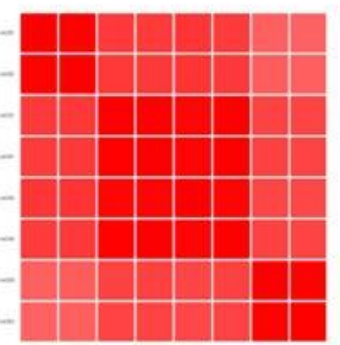

EAS

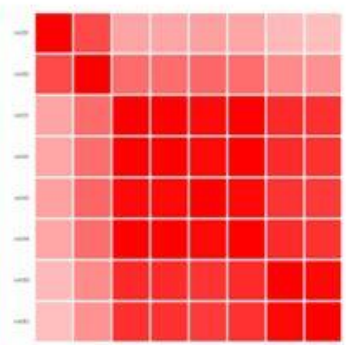

EUR

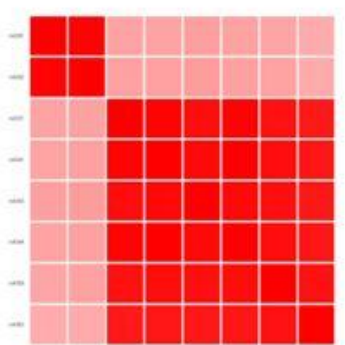

SAS

Figure 2: LD Heatmap plot for ACEAFR- African, AMR- Mixed American, EAS- East Asian, EUR- European, SAS- South Asian

\subsubsection{LD results for gene ESR1:}

The LD heatmap matrix plots for ESR1 gene for different populations were studied and common to all the sample populations of the present study, two SNP variant associations were noticed. SNP variants rs2234693and rs9340799 were noticed to be in high LD along with SNP variants rs4870061 and rs1801132.

\subsubsection{LD results for gene ESR1:}

The LD heatmap matrix plots for MIA3 gene for different populations were studied and common to all the sample populations of the present study, a single SNP variant association was noticed. SNP variants rs10495197 and rs17163303 were noticed to be in high LD.

\subsubsection{Pharmacogenetic Implication Study:}

With a powerful foundation of information related to Linkage Disequilibrium seen in certain SNP variants common to the five sample populations, an advanced implication study related to the Pharmacogenetic association of SNP variant and drugs/chemical was performed utilizing PharmGKB, a Pharmacogenomics research database. According to the Pgx research and previous clinical trials information available from the database, an SNP variant has the potential to influence a drug's therapy either by limiting its efficacy or rendering the drug toxic to the system. For the list of SNP variants found to be associated in linkage disequilibrium were used for the present study. According to the evidence available, the SNP variants could potentially limit the efficacy of certain prescribed drugs for myocardial infarction, coronary heart disease, and other medical conditions.

For the gene ACE, The SNP variant rs4331 was found to influence the drug therapy of prescribed drugs Fluvastatin, Irbesartan, Atenolol. [19] This SNP was associated with two other variants, rs4341, and rs4344 that influence drug therapy for Fluvastatin, Thiazides, Furosemide, Spironolactone, Pravastatin, and Ramipril respectively. [20][21][22] These drugs, especially Fluvastatin, are prescribed for hypercholesterolemia and for prevention of cardiovascular diseases. [23]

For the gene MIA3, with the sole SNP variant association of variants rs10495197 and rs17163303, it was found that each of the SNPs individually has the potential to influence the drug therapy for Vancomycin. [24] Vancomycin is the drug of choice for therapy of bacterial sepsis, meningitis, hemolytic anemia, and for reducing the levels of creatine kinase in blood.
For the gene GATA4, with the sole SNP variant association of variants rs4841588 and rs2645400, it was found that each of the SNPs individually has the potential to influence the drug therapy for Warfarin. [25] Warfarin is an anti-coagulant and is used as a preventive for the formation of blood clots in blood vessels and their migration. [26]

For the gene ESR1, the SNP variant associations of rs9340799 and rs4870061, and rs1801132 and rs2234693 were found to influence the drug therapy of prescribed drugs Letrozole, Raloxifene, Tamoxifen, Atorvastatin, and Anastrozole. [27][28][29] Atorvastatin is a lipid lowering agent and helps in reduction of occurrence of cardiovascular diseases. [30]

Table 1. Annotated SNPs by Gene

\begin{tabular}{|c|c|c|c|}
\hline Gene & SNP & Chemical & Reference \\
\hline \multirow{3}{*}{$\mathrm{ACE}$} & rs4331 & $\begin{array}{l}\text { Atenolol, } \\
\text { Fluvastatin, } \\
\text { Irbesartan }\end{array}$ & [19] \\
\hline & rs 4344 & Ramipril & {$[20]$} \\
\hline & rs 4341 & $\begin{array}{l}\text { Fluvastatin, } \\
\text { Furosemide, } \\
\text { Pravastatin, } \\
\text { Spironolactone, } \\
\text { Thiazides, plain }\end{array}$ & {$[21][22]$} \\
\hline \multirow{2}{*}{ MIA3 } & rs 10495197 & \multirow{2}{*}{ Vancomycin } & \multirow{2}{*}[24]{} \\
\hline & rs 17163303 & & \\
\hline \multirow{2}{*}{ GATA4 } & rs2645400 & \multirow{2}{*}{ Warfarin } & \multirow{2}{*}[25]{} \\
\hline & rs4841588 & & \\
\hline \multirow{4}{*}{ ESR1 } & rs9340799 & $\begin{array}{l}\text { Raloxifene, } \\
\text { Tamoxifen, } \\
\text { Letrozole }\end{array}$ & \multirow[t]{2}{*}[27]{} \\
\hline & rs4870061 & Letrozole & \\
\hline & rs1801132 & $\begin{array}{c}\text { Anastrozole, } \\
\text { Letrozole, } \\
\text { Raloxifene }\end{array}$ & [28] \\
\hline & rs 2234693 & $\begin{array}{c}\text { Anastrozole, } \\
\text { Letrozole, } \\
\text { Atorvastatin }\end{array}$ & [29] \\
\hline
\end{tabular}

\section{CONCLUSION}

In this study, the authors were able to identify genes that are associated with the advent and occurrence of myocardial infarction. Using NCBI's resources and 1000genomes browser, SNP genotype data for SNP variants present on each one of those genes was retrieved. A linkage disequilibrium study of the SNPs was performed to investigate the 
association between two variants which co-inherits them into the progeny. After a firm foundation of LD studies for certain SNP variants, they were subjected to Pharmacogenetic analysis to determine the relationship between the SNP variant and prescribed drugs.

As per the findings of the existence of Linkage Disequilibrium between two unrelated SNP variants on a gene that is associated with the advent of Myocardial Infarction, the authors conclude that these linked variants, for example, rs4331 and rs4341 of ACE gene were inherited together through the progeny from the parent rendering the prescribed drug therapy, in this case, of Fluvastatin and other drugs ineffective. As the study was performed on five different sample populations across the world, there is a high possibility that Fluvastatin may no longer be an effective prescribed medicine for patients with myocardial infarction. Similarly, the results from other SNP variants of other genes indicate that other drugs prescribed for patients with myocardial infarction like Raloxifene, Letrozole, Warfarin, and Vancomycin have a high probability of being ineffective for the next generations in the sample populations. As an alternative course of action, a higher dosage of the drug could be prescribed as compensation. A higher dosage would increase the concentration of the drug available in the system and increase the concentration of the drug that reaches the target tissue. However, increasing the concentration of a drug in the system delivered either orally or intravenously isn't a viable solution. Instead, a better, more practical approach would be to look for alternative medicine, in the form of, medicinal plant extract that has bioactive compounds that share similar structural and therapeutic properties with the listed drugs but are less toxic and have greater bioavailability. Subsequently, further validation of the present study's results through wet lab study will offer a better perspective of the implications of the current study.

The past decade has seen a piqued interest in research advances in pharmacogenomics. Personalized therapy is termed as the future of medicine wherein medicinal therapy and drugs are prescribed to an individual based on their genome data. Linkage Disequilibrium study serves as an ideal foundation for performing pharmacogenetic implication study for a set of haploid genomic data offered for a few sample populations across the world. With a rise in cases of drug therapy ineffectiveness rising across the world, attributed to the differences in the genome, the scope of such research is vast and critical for the further development of personalized medicine.

\section{ACKNOWLEDGMENTS}

The authors thank the management of SNIST for their support and encouragement.

\section{REFERENCES}

[1] Wolf G, Angiotensin II and tubular development. Nephrol Dial Transplant, 2002;17 Suppl 9, 48-51 PMID: 12386287

[2] Ajala AR, Almeida SS, Rangel M, Palomino Z, Strufaldi MW, Puccini RF, Araujo RC, Casarini DE, Franco MC., AR1, 2012. Association of ACE gene insertion/deletion polymorphism with birth weight, blood pressure levels, and ACE activity in healthy children. Am J Hypertens., 2012 Jul; DOI: 10.1038/ajh.2012.50. Epub 2012 May 31., 25(7):827-32

[3] Eloisa Arbustini, Maurizia Grasso, Roberta Fasani, Catherine Klersy, Marta Diegoli, Emanuele Porcu, Nadia
Banchieri, Paolo Fortina, Cesare Danesino, Giuseppe Specchia , A, 1995. Angiotensin converting enzyme gene deletion allele is independently and strongly associated with coronary atherosclerosis and myocardial infarction. Heart - Br Heart J 1995; 74, 584-591. PMCID: PMC484110

[4] Klaus Lindpaintner, M.D., Marc A. Pfeffer, M.D., Ph.D., Reinhold Kreutz, M.D., Meir J. Stampfer, M.D., Dr.P.H., Francine Grodstein, Sc.D., Fran LaMotte, B.S., Julie Buring, Sc.D., and Charles H. Hennekens, M.D., Dr.P.H., A, 1995. A Prospective Evaluation of an Angiotensin-Converting-Enzyme Gene Polymorphism and the Risk of Ischemic Heart Disease. N Engl J Med, 332: March 16, $1995 . \quad$ DOI 10.1056/NEJM199503163321103

[5] Saito K, Chen M, Bard F, Chen S, Zhou H, Woodley D, Polischuk R, Schekman R, Malhotra V., A, 2009. TANGO1 facilitates cargo loading at endoplasmic reticulum exit sites. Cell. 2009, Mar 6;136(5):. DOI: 10.1016/j.cell.2008.12.025., 891-902.

[6] Samani NJ, Erdmann J, Hall AS, Hengstenberg C, Mangino M, Mayer B, Dixon RJ, Meitinger T, Braund P, Wichmann HE, Barrett JH, König IR, Stevens SE, Szymczak S, Tregouet DA, Iles MM, Pahlke F, Pollard H, Lieb W, Cambien F, Fischer M, Ouwehand W, Blankenberg S, Balmforth AJ, Baessler A, Ball SG, Strom TM, Braenne I, Gieger C, Deloukas P, Tobin MD, Ziegler A, Thompson JR, Schunkert H; WTCCC and the Cardiogenics Consortium., A, 2007. T Genome wide association analysis of coronary artery disease. N Engl J Med. Aug 2;357(5): Epub 2007 Jul 18., 443-53. DOI: 10.1056/NEJMoa072366

[7] Diana Lourençoa, Raja Braunerb, Magda Rybczyńskaa, Claire Nihoul-Fékétéc, Ken McElreaveya, and Anu Bashambooa, A, 2011. Loss-of-function mutation in GATA4 causes anomalies of human testicular development. Proc Natl Acad Sci U S A, Jan 25; 108(4) vol. 108 no. 4. 1597-1602. DOI: 10.1073/pnas.1010257108

[8] Xiang R, Fan LL, Huang H, Cao BB, Li XP, Peng DQ, Xia K., A, 2013. A novel mutation of GATA4 (K319E) is responsible for familial atrial septal defect and pulmonary valve stenosis. Gene. 2013 Oct 26. pii: S0378-1119(13)01423-6. 10.1016/j.gene.2013.10.028

[9] Takgi K, Moriguchi T, Miki Y, Nakamura Y, Watanabe M, Ishida T, Yamamoto M, Sasano H, Suzuki T., A 2014. GATA4 immunolocalization in breast carcinoma as a potent prognostic predictor. Cancer Sci., May; 105(5): DOI: $10.1111 /$ cas.12382.

[10] Imai Y. A, 2013. Regulation of bone metabolisms by estrogen/estrogen receptors signaling. Clin Calcium. , Nov; 23(11): DOI: CliCa131116211626. 1621-6.

[11] TWeiyi Toy, Yang Shen, Helen Won, Bradley Green, Rita A Sakr, Marie Will,Zhiqiang Li, Kinisha Gala, Sean Fanning, Tari A King, Clifford Hudis, David Chen,Tetiana Taran, Gabriel Hortobagyi, Geoffrey Greene, Michael Berger, José Baselga \& Sarat Chandarlapaty, A, 2013. ESR1 ligand-binding domain mutations in hormone-resistant breast cancer. Nature Genetics, 45, DOI: 10.1038/ng.2822, 1439-1445. 
[12] Wei CD, Zheng HY, Wu W, Dai W, Tong YQ, Wang M, Li Y., A, 2013. Meta-analysis of the association of the rs2234693 and rs9340799 polymorphisms of estrogen receptor alpha gene with coronary heart disease risk in Chinese Han population. Int J Med Sci., 10(4): DOI: 10.7150/ijms.5234. Epub 2013 Mar 2., 457-66.

[13] Mahmoodzadeh S, Eder S, Nordmeyer J, Ehler E, Huber O, Martus P, Weiske J, Pregla R, Hetzer R, RegitzZagrosek V., 2006. Estrogen receptor alpha upregulation and redistribution in human heart failure. FASEB J, May;20(7), 926-34. DOI: 10.1096/fj.05$5148 \mathrm{com}$

[14] Manolio TA, Collins FS. The HapMap and GenomeWide Association Studies in Diagnosis and Therapy. Annual review of medicine. 2009;60:443-456. DOI :10.1146/annurev.med.60.061907.093117.

[15] Slatkin, Montgomery, Linkage disequilibrium understanding the evolutionary past and mapping the medical future, Nat Rev Genet, 2008/06//print 96477485, Nature Publishing Group, 1471-0056, DOI: $10.1038 / \mathrm{nrg} 2361$

[16] The 1000 Genomes Project Consortium. An integrated map of genetic variation from 1,092 human genomes. Nature. 2012;491(7422):56-65. DOI: 10.1038 /nature 11632

[17] Machiela MJ, Chanock SJ. LDlink a web-based application for exploring population-specific haplotype structure and linking correlated alleles of possible functional variants. Bioinformatics. 2015 Jul 2. PMID: 26139635. DOI: 10.1093/bioinformatics/btv402

[18] M. Whirl-Carrillo, E.M. McDonagh, J. M. Hebert, L. Gong, K. Sangkuhl, C.F. Thorn, R.B. Altman and T.E. Klein. "Pharmacogenomics Knowledge for Personalized Medicine" Clinical Pharmacology \& Therapeutics (2012) 92(4): 414-417. DOI: 10.1038/clpt.2012.96

[19] Thompson JF, Man M, Johnson KJ, Wood LS, Lira ME, Lloyd DB, et al. An association study of 43 SNPs in 16 candidate genes with atorvastatin response. Pharmacogenomics Journal 2005;5(6):352-8. DOI: 10.1038/sj.tpj.6500328

[20] Bozkurt et al, Genetic variation in the renin-angiotensin system modifies the beneficial effects of ACE inhibitors on the risk of diabetes mellitus among hypertensives, J Hum Hypertens, 2008/06/19/online/2211774780, Macmillan Publishing Group 0950-9240, DOI: 10.1038/jhh.2008.62

[21] Suchy-Dicey A, Heckbert SR, Smith NL, et al. Gene expression in thiazide diuretic or statin users in relation to incident type 2 diabetes. International Journal of Molecular Epidemiology and Genetics. 2014;5(1):22-30. [PMCID: PMC3939004]
[22] Bhatnagar V, O'Connor DT, Schork NJ, et al. Angiotensin-converting enzyme gene polymorphism predicts the time-course of blood pressure response to angiotensin converting enzyme inhibition in the AASK trial. Journal of hypertension. 2007;25(10):2082-2092. DOI: 10.1097/HJH.0b013e3282b9720e.

[23] Serruys PWJC, de Feyter P, Macaya C, Kokott N, Puel J, Vrolix M, Branzi A, Bertolami MC, Jackson G, Strauss B, Meier B, for the Lescol Intervention Prevention Study (LIPS) Investigators. Fluvastatin for Prevention of Cardiac Events Following Successful First Percutaneous Coronary Intervention, A Randomized Controlled Trial. JAMA. 2002;287(24):3215-3222. DOI $: 10.1001 /$ jama.287.24.3215.

[24] Van Driest SL, McGregor TL, Velez Edwards DR, et al. Genome-Wide Association Study of Serum Creatinine Levels during Vancomycin Therapy. Thameem F, ed. PLoS ONE. 2015;10(6):e0127791. DOI 10.1371/journal.pone.0127791.

[25] Jeong, E et al, Impact of GATA4 variants on stable warfarin doses in patients with prosthetic heart valves, Pharmacogenomics J, 2015/02//print 1513337, Macmillan Publishers Limited, 1470-269X, DOI: 10.1038/tpj.2014.36

[26] Patel, Manesh R., et al. "Rivaroxaban versus warfarin in nonvalvular atrial fibrillation." New England Journal of Medicine 365.10 (2011): 883-891.

[27] Wang J, Lu K, Song Y, Xie L, Zhao S, et al. (2013) Indications of Clinical and Genetic Predictors for Aromatase Inhibitors Related Musculoskeletal Adverse Events in Chinese Han Women with Breast Cancer. PLoS ONE 8(7): e68798. DOI: 10.1371/journal.pone.0068798

[28] Onitilo, Adedayo A. et al, Estrogen receptor genotype is associated with risk of venous thromboembolism during tamoxifen therapy, Breast Cancer Research and Treatment,1573-7217, http://dx.doi.org/10.1007/s10549008-0264-2

[29] Steffi Oesterreich, N. Lynn Henry, Kelley M. Kidwell, Catherine H. Van Poznak, et al. "Associations between Genetic Variants and the Effect of Letrozole and Exemestane on Bone Mass and Bone Turnover" Breast Cancer Research and Treatment Vol. 154 Iss. 2 (2015) p. 263 - 273 ISSN: 01676806 DOI: 10.1007/s10549-015$3608-8$

[30] Sever, Peter S., et al. "Prevention of coronary and stroke events with atorvastatin in hypertensive patients who have average or lower-than-average cholesterol concentrations, in the Anglo-Scandinavian Cardiac Outcomes Trial-Lipid Lowering Arm (ASCOT-LLA): a multicentre randomised controlled trial." The Lancet 361.9364 (2003): 1149-1158. DOI: $10.1016 / \mathrm{S} 0140$ 6736(03)12948-0 